\title{
Systemic inflammatory response syndrome (SIRS) after extracorporeal membrane oxygenation (ECMO): Incidence, risks and survivals.
}

\author{
Karthik Thangappan \\ Thomas Jefferson University \\ Nicholas C. Cavarocchi \\ Thomas Jefferson University \\ Michael Baram \\ Thomas Jefferson University \\ Brandi Thoma \\ Fbliowathilefferssonditidikersititbrks at: https://jdc.jefferson.edu/surgeryfp \\ (Pashi bfirbseulmonology Commons, and the Surgery Commons \\ Themas is feferso Uwiversitw access to this document benefits you
}

\section{Recommended Citation}

Thangappan, Karthik; Cavarocchi, Nicholas C.; Baram, Michael; Thoma, Brandi; and Hirose, Hitoshi, "Systemic inflammatory response syndrome (SIRS) after extracorporeal membrane oxygenation (ECMO): Incidence, risks and survivals." (2016). Department of Surgery Faculty Papers. Paper 144.

https://jdc.jefferson.edu/surgeryfp/144

This Article is brought to you for free and open access by the Jefferson Digital Commons. The Jefferson Digital Commons is a service of Thomas Jefferson University's Center for Teaching and Learning (CTL). The Commons is a showcase for Jefferson books and journals, peer-reviewed scholarly publications, unique historical collections from the University archives, and teaching tools. The Jefferson Digital Commons allows researchers and interested readers anywhere in the world to learn about and keep up to date with Jefferson scholarship. This article has been accepted for inclusion in Department of Surgery Faculty Papers by an authorized administrator of the Jefferson Digital Commons. For more information, please contact: JeffersonDigitalCommons@jefferson.edu. 
Systemic Inflammatory Response Syndrome (SIRS) after Extracorporeal Membrane Oxygenation (ECMO): Incidence, Risks and Survivals.

None of the authors has a conflict of interest regarding this paper.

Word Count: 2411 


\begin{abstract}
Introduction: Systemic inflammatory response syndrome (SIRS) is frequently observed after extracorporeal membrane oxygenation (ECMO) decannulation; however, these issues have not been investigated well in the past.
\end{abstract}

Methods: Retrospective chart review was performed to identify post-ECMO SIRS phenomenon, defined by exhibiting $2 / 3$ of the following criteria: fever, leukocytosis, and escalation of vasopressors. The patients were divided into 2 groups: patients with documented infections (Group I) and patients with true SIRS (Group TS) without any evidence of infection. Survival and pre-, intra- and post- ECMO risk factors were analyzed.

Results: Among 62 ECMO survivors, 37 (60\%) patients developed the post-ECMO SIRS phenomenon, including Group I $(n=22)$ and Group TS $(n=15)$. The 30-day survival rate of Group I and TS was 77\% and $100 \%$, respectively $(\mathrm{p}=0.047)$, although pre-decannulation risk factors were identical.

Conclusions: SIRS phenomenon after ECMO decannulation commonly occurs. Differentiating between the similar clinical presentations of SIRS and infection is important and will impact clinical outcomes.

Key words: ECMO; SIRS; infection. 


\section{List of Abbreviations}

APACHE: acute physiology and chronic health evaluation.

ECMO: extracorporeal membrane oxygenation.

E-CPR: extracorporeal membrane oxygenation assisted cardiopulmonary resuscitation.

MELD: model for end-stage liver disease.

SAPS: simplified acute physiology score.

SIRS: systemic inflammatory response syndrome.

WBC: white blood cell. 


\section{Introduction}

Extracorporeal membrane oxygenation (ECMO) support is well-established technology that provides both circulatory and respiratory support in patients with severe refractory cardiopulmonary failure. ${ }^{1}$ ECMO stabilizes hemodynamics quickly in patients with severe hemodynamic and respiratory instability. While hemodynamics are restored and stabilized by ECMO, end-organ function is allowed to recover. ${ }^{2}$ Patients on ECMO are decannulated once organ recovery criteria are met. If cardiac recovery has not occurred, the patient can be assessed for implantable mechanical support.

Systemic inflammatory response syndrome (SIRS), including fever, leukocytosis, and vasodilation is often observed during ECMO and related to either the ECMO circuit and/or the patient disease processes. ${ }^{3}$ This SIRS phenomenon during ECMO generally resolves as the primary disease is treated; the SIRS phenomenon should always be absent at the time of decannulation. However, we have often encountered the SIRS phenomenon after ECMO decannulation. The long-standing cannula and extracorporeal circuit kept for a period of time during ECMO may be responsible for blood stream infections. ${ }^{4}$ Similarly, long-term ventilator support may increase the chance of ventilator associated pneumonia and subsequent development of sepsis. ${ }^{4}$ Additionally, surgical interventions required for repair of the vessel after decannulation may complicate the postoperative course. Understanding the nature of the post-decannulation SIRS phenomenon and differentiating the similar clinical presentations of SIRS from sepsis could be important for clinicians who manage ECMO patients, since this can affect patient outcomes. Although we frequently observe these SIRS phenomena after ECMO decannulation, to our knowledge, no research about this issue has been undertaken. Thus, we performed this retrospective study in order to 1) identify the incidence of post-decannulation SIRS, 2) find the incidence of infection, 3) investigate the possibility of differentiating SIRS from infection, and 4) study the outcome of patients based on the presence of SIRS or infection.

\section{Methods}

Between July 2010 and June 2014, 122 ECMO procedures via peripheral cannulation were performed at our institution. ${ }^{4,5}$ Medical records of patients who survived ECMO were reviewed, and 
their pre-, intra-, and post-ECMO data were entered into a structured database after approval from the institutional review board. Patients who died within 48 hours of ECMO decannulation were excluded from the study.

Veno-arterial ECMO from femoral cannulation was used for patients with cardiac failure. ${ }^{4}$ Patients with respiratory failure underwent veno-venous ECMO via single right internal jugular cannulation, ${ }^{5}$ although a minority of these patients underwent veno-arterial ECMO due to anatomy, severe hypotension, ventricular failure, or technical reasons. ECMO circuits included a Rotaflow centrifugal pump (Maquet, Rastatt, Germany) and a Quadrox-D diffusion membrane hollow-fiber oxygenator (Maquet, Rastatt, Germany). All patients were afebrile without evidence of on-going active infection or SIRS at the time of decannulation, per our institutional practice guidelines. ECMO decannulation was performed in the operating room with direct vessel repair. ${ }^{4,5}$ Peri-operative antibiotics were used for only 24 hours post-decannulation unless a course of antibiotics initiated during ECMO were being completed. Antibiotics were extended past 24 hours post-decannulation based on clinical judgment if a patient exhibited signs of a new infection.

Post-ECMO decannulation "SIRS phenomenon" was defined as having 2 out of the 3 of the following criteria after ECMO decannulation regardless of the presence of infection: fever (temperature > $101.5^{\circ} \mathrm{F}$ ), leukocytosis (white blood cell $[\mathrm{WBC}]>12,000$, or $25 \%$ increase from pre-decannulation baseline), and escalation of vasopressors compared to the patient's pre-decannulation baseline. Other diagnostic criteria of the SIRS, such as increase in heart rate or respiratory rate were not used for this study since these may be artificially modified due to the use of inotropes, degree of sedation, and ventilator settings. The patients who were found to have the SIRS phenomenon were further divided into 2 groups: SIRS phenomenon with evidence of infection (Group I) and true SIRS without evidence of infection (Group TS). Evidence of infection included positive blood, sputum, wound, or stool cultures. The presence of the infection was divided into "carried over" which was defined as infection present at the time of cannulation or during ECMO, but controlled appropriately at the time of the decannulation, 
and "new infection after ECMO decannulation" which was defined as culture positive infection diagnosed after ECMO decannulation. Figure 1 illustrates the division of patient groups used in the study.

Risk factors including patient demographics, pre-ECMO, intra-ECMO, and post-ECMO parameters were retrospectively analyzed to identify risk factors of developing the post-decannulation SIRS phenomenon and variables that can differentiate between post-decannulation infection and true SIRS. Categorical variables and continuous variables were compared between the two groups using ChiSquare analysis (Fisher tests if small sample size) or two-tailed t-test, as appropriate. A p-value of less than 0.05 was considered to be significant.

\section{Results}

A total of 62 patients who survived ECMO were identified during the study period. Among them, 37 (60\%) patients developed the SIRS phenomenon based on the criteria. Table 1 compared the demographics of the patients with post-decannulation SIRS phenomenon vs. no SIRS phenomenon. There were no significant differences in pre-ECMO risk factors, hematology, or chemistry values except for following: pre-ECMO lactate $(4.8 \pm 4.6 \mathrm{mmol} / \mathrm{L}$ for the patients with SIRS phenomenon vs. $2.9 \pm 2.4$ $\mathrm{mmol} / \mathrm{L}$ without SIRS phenomenon, $\mathrm{p}=0.038)$ and alanine aminotransferase (93 $\pm 190 \mathrm{IU} / \mathrm{L}$ vs. $288 \pm 440$ IU/L, p=0.041). Pre-decannulation demographics, pre-decannulation laboratory data, and length of ECMO were similar between those who developed post-decannulation SIRS phenomenon and those who did not. Similarly, complications during ECMO and transfusions during ECMO (15 \pm 20 units packed blood cell transfusion for those with SIRS phenomenon vs. $16 \pm 18$ units for those without SIRS phenomenon) were not significantly different. The 30-day survival after ECMO decannulation was not different between with and without post-decannulation SIRS phenomenon (86\% with the SIRS phenomenon vs. $76 \%$ without the SIRS phenomenon, $\mathrm{p}=0.289)$.

Among those who had post-decannulation SIRS phenomenon, 22 patients (59\% among SIRS phenomenon patients, 35\% among all studied patients) had evidence of infection (Group I): bacteremia in $7(32 \%)$, pneumonia in $12(56 \%)$, surgical site infection in $3(14 \%)$, and Clostridium difficile infection in $1(4.5 \%)$, with 5 patients having overlapping infections. Out of these 22 patients, 9 patients $(41 \%)$ had 
carried over an infection that developed either at the time of ECMO cannulation or during ECMO, although these infections had been controlled with appropriate antibiotics. The remaining 13 patients (59\%) had infections that developed after decannulation. In Group TS, which included 15 patients (41\% among SIRS phenomenon patients, 24\% among all studied patients), no evidence of infection was found despite aggressive investigation of possible sources of infection including serial cultures and imaging tests. Demographics of Group I and Group TS are shown in Table 2. Risk factor analysis showed that metabolic acidosis at the time of ECMO cannulation was less frequently observed in Group I than in Group TS (Group I vs. Group TS: pH $7.32 \pm 0.12$ vs. pH $7.23 \pm 0.11, \mathrm{p}=0.031$; and $\mathrm{HCO}_{3} 23 \pm 6 \mathrm{mmol} / \mathrm{L}$ vs. $\mathrm{HCO}_{3} 19 \pm 5 \mathrm{mmol} / \mathrm{L}, \mathrm{p}=0.038$ respectively). Pre-ECMO demographics, pre-ECMO laboratory data, length of ECMO, pre-decannulation laboratory data, complications during ECMO, and transfusions during ECMO were not different between Group I and Group TS.

Individual components of the SIRS phenomenon after decannulation of ECMO are shown in Table 3. Development of fever (initial day of fever, length of fever), leukocytosis (initial day of leukocytosis, duration of leukocytosis), and vasopressor usage (number of patients requiring vasopressor escalation, duration of vasopressor use) were similar between Group I and Group TS. In comparing variables of SIRS phenomenon between the two groups, the only difference found was the peak WBC count (Group I vs. Group TS: $30.1 \pm 13.3$ B/L vs. $21.3 \pm 7.7$ B/L, p=0.04). Peak WBC counts above 30,000 were more often found in Group I (46\%) than in Group TS (14\%), p=0.026. Regardless of the presence of infection or not, the usage of antibiotics beyond the 24-hour prophylaxis in patients who had post-decannulation SIRS phenomenon was similar between Group I and Group TS (antibiotics usage $68 \%$, length of use $8.7 \pm 6.1$ days in Group I vs. antibiotics usage $53 \%$, length of use $6.5 \pm 4.9$ days in Group TS).

A small number of the patients had procalcitonin levels measured after ECMO decannulation due to clinical suspicion of infection (22 patients in Group I and 8 patients in Group TS). The mean procalcitonin was $3.3 \pm 1.8 \mathrm{ng} / \mathrm{dl}$ in Group I and $1.8 \pm 1.7 \mathrm{ng} / \mathrm{dl}$ in Group TS, $\mathrm{p}=0.2552$. The trend of higher procalcitonin values was observed more in Group I than in Group TS. 
The post-decannulation 30-day survival rate was lower in Group I $(77 \%, 17 / 22)$ than in Group TS $(100 \%, 15 / 15)$ with $\mathrm{p}=0.047$. Of the five patients in Group I who died within 30 days, three of those deaths were related to post-decannulation infection (two due to infection related to permanent left ventricular assist device implantation and subsequent open chest management unrelated to ECMO, one due to severe pneumonia which developed after ECMO decannulation), and the other two were related to cardiac arrest secondary to possible in-stent thrombosis after ECMO removal. The survival of those who had post-decannulation infection carrying over from ECMO was $78 \%$ (7/9), which was similar to that of those who developed new infections after ECMO removal (77\%, 10/13).

Based on the SIRS phenomenon criteria, there were 25 patients who did not have the SIRS phenomenon. Among these patients without SIRS phenomenon, 10 patients (40\%) had evidence of infection and 15 patients (60\%) had no evidence of infection. The survival rates were $60 \%$ and $87 \%$, respectively $(\mathrm{p}=0.126)$.

\section{Discussion}

This study showed a frequent incidence of the SIRS phenomenon including fever, elevation of WBC, and hypotension requiring vasopressors after decannulation of ECMO, even though none of these patients have the SIRS phenomenon before decannulation of ECMO. Almost all patients developed one of the three criteria of the post-decannulation SIRS phenomenon, and two-thirds of the patients fell under our definition of the SIRS phenomenon. Although risk factor analyses showed pre-ECMO high lactate and low alanine aminotransferase were associated with development of the SIRS phenomenon after ECMO decannulation, we believe this pre-ECMO metabolic disarrangement most likely does not contribute an essential role to the development of the SIRS phenomenon since these levels were normalized before decannulation (Table 1). Thus, the risk factors for development of the postdecannulation SIRS phenomenon remain undetermined.

Among those who had the SIRS phenomenon, the patterns of fever, leukocytosis and vasopressor requirements were almost identical between those who had infection and those who did not, although the outcomes were significantly inferior in the patients who had infections compared to those who had true 
SIRS. Once patients developed the SIRS phenomenon after ECMO decannulation, it could continue for almost a week regardless of the presence of infection. However, a survival difference was clear based on the presence of infection. The only difference in the SIRS phenomenon criteria between Group I and TS was the highest WBC count. Patients with infection developed higher WBC counts; although patients with true SIRS also had elevated WBC count. Although leukocytosis is not specific to infection, a WBC count greater than 30,000 is more likely infectious in etiology. Extensive work up is necessary in these patients, and empiric antibiotics are recommended.

The high incidence of the post-ECMO decannulation SIRS phenomenon is unique and has not previously been noted in the literature. Although Aubron et al. demonstrated that bleeding is a frequent complication of ECMO, ${ }^{6}$ our study showed that neither bleeding events or transfusion requirement did not significantly impact the development of SIRS or infections after ECMO decannulation.

Peri-operative antibiotics were used for 24 hours after ECMO decannulation, unless the patient had ongoing treatment for infection during or prior to the ECMO period. Identifying the infection shortly after ECMO decannulation may be challenging. Our study showed that among those who had the SIRS phenomenon, more than half $(\sim 60 \%)$ of the patients had a significant infection, which affected the 30 -day survival. Patients with the SIRS phenomenon with a suspected infection should be treated aggressively with broad-spectrum antibiotics until culture results are available. Due to lack of clear clinical differentiation between infection and the SIRS phenomenon, empiric antibiotics were often employed in these patients. This may potentially increase costs and antimicrobial resistance; however, we did not analyze these variables.

Although infection is a risk factor for post-decannulation death, it may be difficult to identify infections since finalized culture results may take up to 5 days. Since 2012, we have used the procalcitonin level as a part of the work up for infection in conjunction with routine cultures. ${ }^{7}$ Procalcitonin is a biomarker of infection and has been reported to be more specific than WBC count or Creactive protein. Based on our previous study, ${ }^{7}$ the procalcitonin level used to identify infection was 2 ng/dl. ${ }^{7}$ Among patients who had a procalcitonin level measured after ECMO decannulation, we found a 
trend of higher procalcitonin values in Group I than in Group TS, although the sample size was too small to conclude statistical significance. Since the procalcitonin value can be obtained as a point of care assay within hours of collection of the sample, procalcitonin may have a role in the early prediction of infection in patients with SIRS phenomenon after decannulation of ECMO.

This study has limitations due to a small sample size, single institution practice, and retrospective nature. Identification of infection was also a limitation. We defined infection as the presence of positive culture results; however, the culture results may be dependent on the use of antibiotics. The use of antibiotics in patients with the SIRS phenomenon was determined by the clinical judgment of a multidisciplinary intensive care team with infectious disease consultation. Since post-decannulation infection increased the mortality in this study, routine prolonged courses of broad-spectrum antibiotics could be considered to minimize the risk of the post-decannulation infection, although this incidence of surgical site infection in this study was minimal. Future multi-centered studies with larger patient populations would be necessary to further investigate this post-ECMO SIRS phenomenon, along with standardizing decannulation techniques, assessment of infection, and antimicrobial management strategies.

\section{Conclusions}

Both post-ECMO SIRS phenomenon ( $60 \%)$ and post-decannulation infection ( $60 \%$ including infection carried over during ECMO, and 35\% newly developed infection after ECMO decannulation) were common in our single institution experience. The differentiation of infection from true SIRS was difficult and remains challenging. The outcomes of those who had infection after ECMO decannulation were poor, while $100 \%$ of true SIRS patients survived. The SIRS phenomenon after ECMO decannulation should be treated as infection until proven otherwise in order to optimize hospital outcomes. 
Table 1: Demographics of patients with and without SIRS phenomenon. Data are expressed as number $(\%)$ or mean \pm standard deviation.

\begin{tabular}{|c|c|c|c|}
\hline & $\begin{array}{c}\text { SIRS } \\
\text { Phenomenon } \\
n=37\end{array}$ & $\begin{array}{c}\text { No SIRS } \\
\text { Phenomenon } \\
n=25\end{array}$ & p-value \\
\hline \multicolumn{4}{|l|}{ Pre-ECMO Demographics } \\
\hline Age (years) & $48 \pm 13$ & $47 \pm 15$ & 0.787 \\
\hline Male gender & $29(78 \%)$ & $15(60 \%)$ & 0.118 \\
\hline Cardiac ECMO & $24(65 \%)$ & $12(48 \%)$ & 0.187 \\
\hline Respiratory ECMO & $13(35 \%)$ & $13(52 \%)$ & 0.187 \\
\hline E-CPR & $5(14 \%)$ & $5(20 \%)$ & 0.496 \\
\hline \multicolumn{4}{|l|}{ Clinical diagnoses } \\
\hline Cardiogenic Shock & $25(68 \%)$ & $12(48 \%)$ & 0.123 \\
\hline Post-cardiotomy failure & $5(14 \%)$ & $1(4.0 \%)$ & 0.214 \\
\hline Acute respiratory distress syndrome & $10(27 \%)$ & $10(40 \%)$ & 0.284 \\
\hline \multicolumn{4}{|l|}{ Pre-ECMO laboratory data } \\
\hline White blood cell count (B/L) & $13.1 \pm 6.9$ & $14.3 \pm 7.7$ & 0.533 \\
\hline Creatinine $(\mathrm{mg} / \mathrm{dl})$ & $1.5 \pm 0.9$ & $1.4 \pm 0.8$ & 0.648 \\
\hline Bilirubin (mg/dl) & $1.4 \pm 1.6$ & $1.3 \pm 1.2$ & 0.780 \\
\hline Aspartate aminotransferase (IU/L) & $160 \pm 190$ & $290 \pm 419$ & 0.151 \\
\hline Alanine aminotransferase (IU/L) & $93 \pm 190$ & $288 \pm 440$ & 0.041 \\
\hline Lactate $(\mathrm{mmol} / \mathrm{L})$ & $4.8 \pm 4.6$ & $2.9 \pm 2.4$ & 0.038 \\
\hline \multicolumn{4}{|l|}{ Pre-ECMO Scores } \\
\hline SAPS II & $53.6 \pm 15.1$ & $56.5 \pm 19.6$ & 0.534 \\
\hline APACHE II & $28.2 \pm 8.3$ & $27.8 \pm 7.3$ & 0.842 \\
\hline MELD & $15.6 \pm 7.6$ & $13.7 \pm 6.2$ & 0.285 \\
\hline \multicolumn{4}{|l|}{ Complication during ECMO } \\
\hline Stroke & $2(5 \%)$ & $0(0 \%)$ & 0.237 \\
\hline Pneumonia & $8(22 \%)$ & $4(16 \%)$ & 0.583 \\
\hline \multicolumn{4}{|l|}{ Pre-Decannulation laboratory data } \\
\hline White blood cell count $(\mathrm{B} / \mathrm{L})$ & $16.2 \pm 6.1$ & $20 \pm 8.9$ & 0.096 \\
\hline Platelet count $(\mathrm{B} / \mathrm{L})$ & $124 \pm 63$ & $130 \pm 61$ & 0.735 \\
\hline Creatinine (mg/dl) & $1.2 \pm 0.5$ & $1.2 \pm 0.9$ & 1.000 \\
\hline Bilirubin $(\mathrm{mg} / \mathrm{dl})$ & $2.6 \pm 3.2$ & $1.7 \pm 1.2$ & 0.125 \\
\hline Aspartate aminotransferase (IU/L) & $68 \pm 41$ & $72 \pm 89$ & 0.834 \\
\hline Alanine aminotransferase (IU/L) & $71 \pm 71$ & $72 \pm 62$ & 0.953 \\
\hline Lactate $(\mathrm{mmol} / \mathrm{L})$ & $1.5 \pm 0.5$ & $1.8 \pm 1.8$ & 0.439 \\
\hline Length of ECMO (days) & $10 \pm 5.1$ & $8.8 \pm 5.3$ & 0.378 \\
\hline \multicolumn{4}{|l|}{ Outcomes } \\
\hline 30-day survival & $32(86 \%)$ & $19(76 \%)$ & 0.289 \\
\hline \multicolumn{4}{|l|}{ SIRS Phenomenon } \\
\hline Fever & $35(95 \%)$ & $17(68 \%)$ & 0.005 \\
\hline Leukocytosis & $27(73 \%)$ & $4(16 \%)$ & 0.000 \\
\hline Escalation of vasopressors & $30(81 \%)$ & $11(44 \%)$ & 0.003 \\
\hline Infection & $22(59 \%)$ & $10(40 \%)$ & 0.133 \\
\hline
\end{tabular}


Table 2: Demographics of patients for Group I (Infection group) and Group TS (True SIRS group). Data are expressed as number $(\%)$ or mean \pm standard deviation.

\begin{tabular}{|c|c|c|c|}
\hline & $\begin{array}{c}\text { Group I } \\
\mathrm{N}=22\end{array}$ & $\begin{array}{c}\text { Group TS } \\
\mathrm{N}=15\end{array}$ & p-value \\
\hline \multicolumn{4}{|l|}{ Pre-ECMO Demographics } \\
\hline Age (years) & $51 \pm 14$ & $45 \pm 13$ & 0.222 \\
\hline Male gender & $18(82 \%)$ & $11(73 \%)$ & 0.538 \\
\hline Cardiac ECMO & $15(68 \%)$ & $9(60 \%)$ & 0.609 \\
\hline Respiratory ECMO & $7(32 \%)$ & $6(40 \%)$ & 0.609 \\
\hline E-CPR & $4(18 \%)$ & $1(6.7 \%)$ & 0.314 \\
\hline \multicolumn{4}{|l|}{ Clinical risk factors } \\
\hline Coronary artery disease & $12(55 \%)$ & $4(27 \%)$ & 0.093 \\
\hline Post-cardiotomy failure & $2(9.1 \%)$ & $3(20 \%)$ & 0.341 \\
\hline Acute respiratory distress syndrome & $6(27 \%)$ & $4(27 \%)$ & 0.968 \\
\hline \multicolumn{4}{|l|}{ Pre-ECMO laboratory data } \\
\hline White blood cell count (B/L) & $14.5 \pm 8$ & $11.1 \pm 4.3$ & 0.104 \\
\hline $\mathrm{HCO}_{3}(\mathrm{mmol} / \mathrm{L})$ & $23 \pm 6$ & $19 \pm 5$ & 0.038 \\
\hline Creatinine (mg/dl) & $1.6 \pm 0.8$ & $1.4 \pm 1.0$ & 0.522 \\
\hline Bilirubin $(\mathrm{mg} / \mathrm{dl})$ & $1.5 \pm 1.6$ & $1.4 \pm 1.7$ & 0.866 \\
\hline Aspartate aminotransferase (IU/L) & $134 \pm 196$ & $189 \pm 376$ & 0.615 \\
\hline Alanine aminotransferase (IU/L) & $67 \pm 71$ & $123 \pm 269$ & 0.440 \\
\hline Lactate $(\mathrm{mmol} / \mathrm{L})$ & $4.6 \pm 5.2$ & $5.0 \pm 3.7$ & 0.795 \\
\hline \multicolumn{4}{|l|}{ Pre-ECMO Scores } \\
\hline SAPS II & $55 \pm 15$ & $52 \pm 15$ & 0.558 \\
\hline APACHE II & $28 \pm 7$ & $29 \pm 10$ & 0.752 \\
\hline MELD & $17 \pm 7$ & $14 \pm 8$ & 0.271 \\
\hline \multicolumn{4}{|l|}{ Complication during ECMO } \\
\hline Stroke & $2(9.1 \%)$ & $0(0 \%)$ & 0.230 \\
\hline Pneumonia & $3(14 \%)$ & $2(13 \%)$ & 0.979 \\
\hline \multicolumn{4}{|l|}{ Pre-Decannulation laboratory data } \\
\hline White blood cell count (B/L) & $16.6 \pm 5.8$ & $15.5 \pm 6.7$ & 0.608 \\
\hline Platelet count $(\mathrm{B} / \mathrm{L})$ & $128 \pm 64$ & $120 \pm 63$ & 0.695 \\
\hline Creatinine (mg/dl) & $1.3 \pm 0.5$ & $1.1 \pm 0.6$ & 0.295 \\
\hline Bilirubin $(\mathrm{mg} / \mathrm{dl})$ & $2.5 \pm 3.5$ & $2.6 \pm 2.8$ & 0.924 \\
\hline Aspartate aminotransferase (IU/L) & $60 \pm 34$ & $81 \pm 48$ & 0.153 \\
\hline Alanine aminotransferase (IU/L) & $57 \pm 50$ & $93 \pm 91$ & 0.172 \\
\hline Lactate $(\mathrm{mmol} / \mathrm{L})$ & $1.5 \pm 0.6$ & $1.5 \pm 0.4$ & 1.000 \\
\hline Length of ECMO (days) & $9.8 \pm 4.7$ & $10.7 \pm 5.8$ & 0.621 \\
\hline \multicolumn{4}{|l|}{ Outcomes } \\
\hline 30-day survival & $17(77 \%)$ & $15(100 \%)$ & 0.047 \\
\hline
\end{tabular}


Table 3: Post-decannulation data. Data are expressed as number (\%) or mean \pm standard deviation.

\begin{tabular}{|c|c|c|c|}
\hline & $\begin{array}{c}\text { Group I } \\
n=22\end{array}$ & $\begin{array}{c}\text { Group TS } \\
n=15\end{array}$ & p-value \\
\hline Incidence of fever & $22(100 \%)$ & $13(87 \%)$ & 0.078 \\
\hline First day of fever (days from decannulation) & $1.3 \pm 2.3$ & $0.7 \pm 0.9$ & 0.283 \\
\hline Duration of fever (days) & $7.6 \pm 4.9$ & $6.7 \pm 6.0$ & 0.650 \\
\hline Incidence of leukocytosis (\%) & $16(73 \%)$ & $11(73 \%)$ & 0.968 \\
\hline First day of leukocytosis (days from decannulation) & $1.3 \pm 1.1$ & $1.3 \pm 1.1$ & 1.000 \\
\hline Peak of white blood cell count (B/L) & $30.1 \pm 13.3$ & $21.3 \pm 7.7$ & 0.040 \\
\hline Peak of white blood cell count above $30 \mathrm{k}(\mathrm{B} / \mathrm{L})$ & $7(46 \%)$ & $3(14 \%)$ & 0.026 \\
\hline Duration of leukocytosis (days) & $7.3 \pm 4.5$ & $6.0 \pm 4.2$ & 0.450 \\
\hline Escalation of vasopressors (\%) & $9(41 \%)$ & $7(47 \%)$ & 0.729 \\
\hline Duration of escalation of vasopressors (days) & $5.9 \pm 3.0$ & $4.4 \pm 2.6$ & 0.303 \\
\hline Usage of antibiotics beyond prophylaxis (\%) & $15(68 \%)$ & $8(53 \%)$ & 0.531 \\
\hline Duration of antibiotics beyond prophylaxis (days) & $8.7 \pm 6.1$ & $6.5 \pm 4.9$ & 0.366 \\
\hline
\end{tabular}


Figure 1: Division of patient groups.

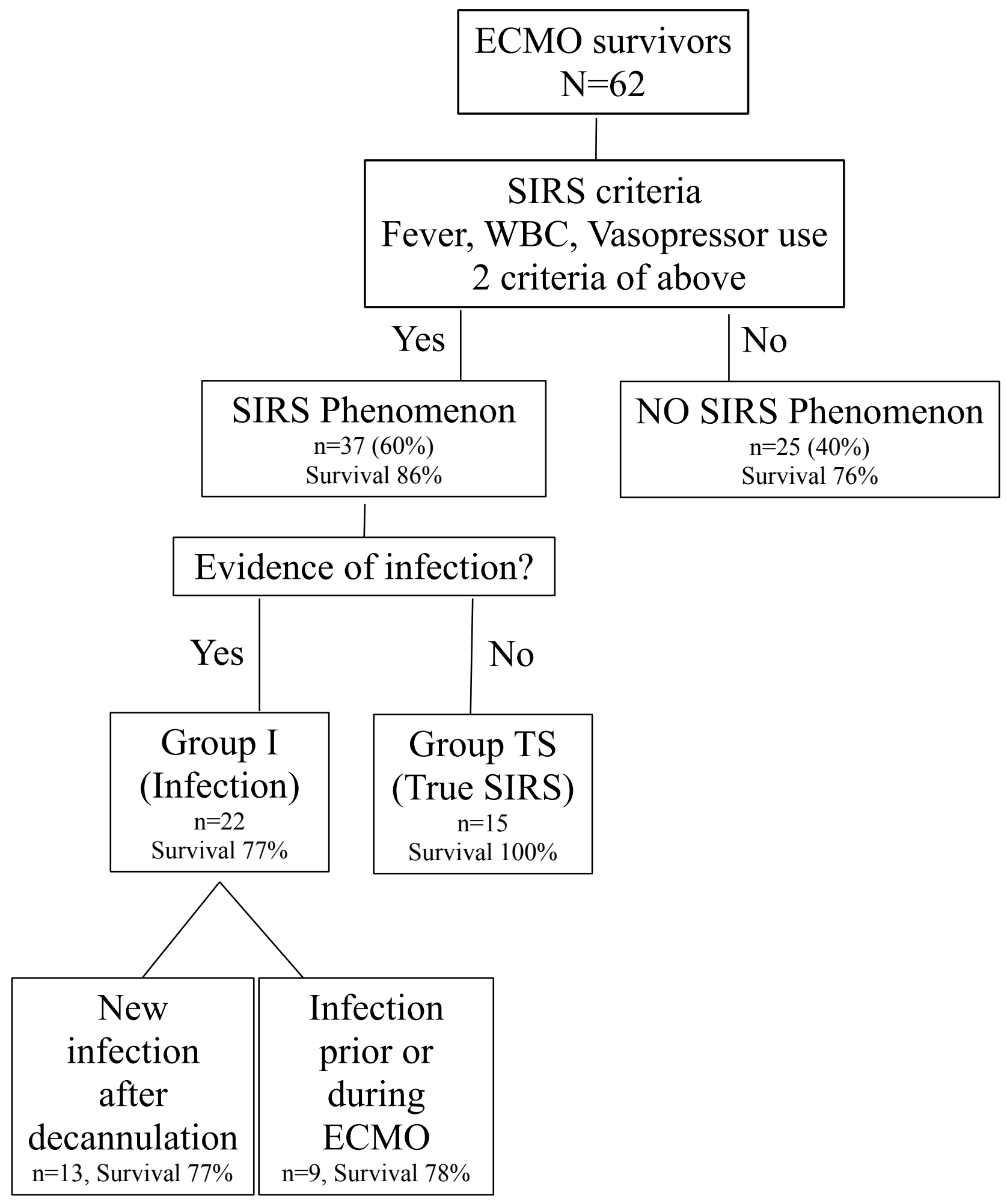




\section{References}

1. Abrams D, Combes A, Brodie D. Extracorporeal membrane oxygenation in cardiopulmonary disease in adults. J Am Coll Cardiol. 2014 ;63:2769-78. doi: 10.1016/j.jacc.2014.03.046.

2. Wong JK, Siow VS, Hirose H, Karbowski P, Miessau J, Baram M, DeCaro M, Pitcher HT, Cavarocchi NC. End organ recovery and survival with the QuadroxD oxygenator in adults on extracorporeal membrane oxygenation. Word J Cardiovasc Surg 2012;2:73-80. doi: 10.4236/wjcs.2012.24015,

3. He C, Yang S, Yu W, Chen Q, Shen J, Hu Y, Shi J, Wu X, Li J, Li N. Effects of continuous renal replacement therapy on intestinal mucosal barrier function during extracorporeal membrane oxygenation in a porcine model. J Cardiothorac Surg 2014 Apr 23;9:72. doi: 10.1186/1749-8090-972.

4. Haneke F, Schildhauer TA, Schlebes AD, Strauch JT, Swol J. Infections and Extracorporeal Membrane Oxygenation: Incidence, Therapy, and Outcome. ASAIO J. 2016;62:80-6. doi: 10.1097/MAT.0000000000000308.

4. Lamb K M, Hirose H, Cavarocchi NC. Preparation and technical considerations for percutaneous cannulation for veno-arterial extracorporeal membrane oxygenation. J Cardiothorac Surg. 2013;28:190-2. doi: 10.1111/jocs.12058.

5. Shaheen A, Tanaka D, Cavarocchi NC, Hirose H. Veno-venous extracorporeal membrane oxygenation (VV ECMO) indications, preprocedural considerations, and technique. J Card Surg 2016; 31:248-252. doi: $10.111 /$ jocs. 12690 .

6. Aubron C, Cheng AC, Pilcher D, Leong T, Magrin G, Cooper DJ, Scheinkestel C, Pellegrino V. Factors associated with outcomes of patients on extracorporeal membrane oxygenation support: A 5year cohort study. Crit Care. 2013;17:R73. doi: 10.1186/cc12681.

7. Tanaka D, Pitcher HT, Cavarocchi NC, Hirose H. Can procalcitonin differentiate infection from systemic inflammatory reaction in patients on extracorporeal membrane oxygenation? J Heart Lung Transplant 2014;33 (11): 1186-1188. doi: 10.1016/j.healun.2014.08.015. 